\title{
AVERSION TO THE VARIABILITY OF PAY AND THE STRUCTURE OF EXECUTIVE COMPENSATION CONTRACTS
}

\author{
Pierre CHAIGNEAU \\ Finance department, HEC Montreal, Montreal, Canada, 3000 Chemin \\ de la Côte Sainte Catherine, Montreal H3T 2A7, QC, Canada \\ E-mail: pierre.chaigneau@hec.ca
}

Received 19 March 2013; accepted 27 August 2014

\begin{abstract}
This paper presents a new implication of an aversion toward the variance of pay ("risk aversion") for the structure of managerial incentive schemes. In a principalagent model in which the effort of a manager with mean-variance preferences affects the mean of a performance measure, we find that managerial compensation must be such that the variance of payments is decreasing in effort. From an ex-ante perspective, which is relevant for effort inducement, this maximizes the rewards associated to high effort, and the punishments associated to low effort. An important practical implication is that convex incentive contracts do not satisfy this necessary condition for optimality, which calls into question the practice of granting executive stock options. The paper therefore contributes to the debate on the efficiency of executive compensation.
\end{abstract}

Keywords: executive compensation, incentives, moral hazard, principal-agent, risk aversion, stock options.

JEL Classification: D86, J33, M52.

\section{Introduction}

The alignment of interests between the manager and the shareholders of a firm is a corporate governance problem of the first order. It is generally studied with principalagent models of moral hazard (e.g., Salanié 1997; Laffont, Martimort 2002; Bolton, Dewatripont 2005). It is well-known that, with a risk-neutral manager and no constraints on contracting, the first-best outcome (without an agency problem) is feasible at the second-best, and the structure of incentives does not matter. In the case of a risk-averse manager, however, when incentive provision implies a deviation from first-best risk sharing, the structure of incentives matters. Even though optimality results can be obtained in certain settings ${ }^{1}$, it remains unclear how the optimal balance between rewards and punishments is determined.

\footnotetext{
${ }^{1}$ For instance, for an exponential distribution and an agent with square root utility (Holmstrom 1979), for a lognormal distribution and an agent with CRRA utility (Dittmann, Maug 2007), for a gamma distribution and an agent with HARA utility (Hemmer et al. 2000).
} 
This paper studies the effect of a manager's aversion toward the variability of his pay for the form of his compensation contract. In order to focus on the effect of risk aversion on contract design, we assume that the manager has mean-variance preferences (Johnstone, Lindley 2011) $)^{2}$. This is both a strength and a limitation of our approach. On the one hand, we can isolate the effect of risk aversion for contract design. On the other hand, we only consider the manager's preferences about the second moment of the probability distribution of future wealth. Our main result is that it is optimal for the equilibrium variance of the manager's pay to be a decreasing function of his effort if effort affects the mean of the performance measure.

In particular, this necessary condition for optimality establishes the suboptimality of convex contracts, i.e., contracts such that the pay-performance sensitivity is increasing in performance, including stock options ${ }^{3}$. The widespread practice of granting options to managers had already been criticized for a number of reasons (Hall, Murphy 2003). This paper identifies a new reason for the suboptimality of stock options, thereby contributing to the debate on the efficiency of managerial compensation (Bebchuk, Fried 2003; Edmans, Gabaix 2009). It can also contribute to explain why executive bonuses are typically capped (Murphy 1999). Finally, a recent literature, starting with Sundaram and Yermack (2007), emphasizes that CEOs hold substantial "inside debt", whose value is sensitive to firm value only in bankruptcy. This paper contributes to explain why inside debt, whose value only varies if performance is below a threshold, may be part of an optimal contract (see also Edmans, Liu 2011).

The intuition behind the paper's main result can be explained as follows. Each manager can be viewed as having a type, namely the probability distribution of performances associated to the level of effort he exerted. The objective of giving adequate incentives while minimizing deviations from the first-best risk sharing rule for the type who exerts high effort is notably achieved by exposing him to a lower variance of pay than the type who exerts low effort. This can be done by using punishments for poor performances (which are unlikely under high effort but more likely under low effort) rather than rewards for good performances. With such an incentive scheme, not only do managers exert higher effort to reduce the variance of their pay, but the variance of pay is especially low for the type who exerts high effort, which minimizes the agency cost of incentive provision. This effect relies crucially on two assumptions: the manager must be risk averse, and his performance must be a noisy version of his effort. In addition, the economic importance of this effect will be greater for more risk averse managers,

\footnotetext{
${ }^{2}$ Postulating a utility function with quadratic terms, i.e. of the form $u(w)=a w-b w^{2}$, implies that maximizing expected utility is equivalent to maximizing a mean-variance criterion of the form $E[u(w)]=a E[w]-b\left(\operatorname{var}(w)+(E[x])^{2}\right)$. By contrast, we consider a more standard linear meanvariance criterion of the form $E[w]-\omega \operatorname{var}(w)$. This is notably the criterion which is maximized by an agent with constant absolute risk aversion (CARA) utility whose wealth is normally distributed. One advantage of this criterion relative to quadratic utility is that the agent's utility is always increasing in his expected compensation, which is not necessarily the case with quadratic utility.

${ }^{3}$ With CRRA-lognormal principal-agent models, the managerial compensation literature also concludes that stock options are suboptimal (Hall, Murphy 2002; Dittmann, Maug 2007).
} 
so that factors such as age and wealth, which affect the manager's risk aversion (Becker 2006), should affect the form of the contract.

Constraints on contracting, especially the limited liability constraint which rules out negative payments to the manager, may explain the discrepancy between theoretically optimal contracts (e.g., Mirrlees 1975) and the contracts observed in practice (Dittmann et al. (2011) also study the effect of constraints on contracting in a model in which the manager is loss averse). To take this into account, we consider step contracts, which are such that the manager is paid a certain amount if performance is below a threshold, and a higher amount if performance is above a threshold. We show that a lower bound on payments such as a limited liability constraint is then binding at the optimum, i.e., the low payment will be as low as possible. The intuition is once again that using punishments for incentive purposes makes the variance of pay decreasing in effort, which increases effort incentives and reduces the agency cost.

We then consider the case in which effort affects both the mean and the variance of the performance measure. Whereas convex contracts remain suboptimal if the variance of the performance measure is nonincreasing in effort, this result does not necessarily hold otherwise. In particular, for an approximately risk neutral manager, concave contracts are now suboptimal if the variance of the performance measure is increasing in effort. Indeed, in this case, convex contracts provide more effort incentives because the slope of the contract covaries positively with the effect of effort on the performance measure. With an approximately risk neutral manager, this effect dominates the effect already mentioned. Thus, the model predicts that managers who innovate rather than exploit existing technologies (in the terminology of Manso (2011)) will have more convex compensation profiles, ceteris paribus. This is consistent with the evidence in Coles et al. (2006) and Francis et al. (2011).

Our results have implications for the measurement of managerial incentives. We argue that the average pay-performance sensitivity, which does not account for the incentive effect of the structure of executive compensation (whether "rewards" or "punishments" are primarily used), does not accurately measure the incentives of risk averse managers.

There are three types of papers that analyze the structure of executive compensation with risk averse CEOs. First, some papers use the expected utility approach but assume a given contract which is linear in the performance measure(s) (e.g., Gibbons, Murphy 1992; Holmstrom, Tirole 1993; Garen 1994), so that they do not study the effect of risk aversion on the structure of the optimal contract. Second, some papers use the expected utility approach and derive the form of the optimal contract (e.g., Hemmer et al. 2000; Dittmann, Maug 2007; Dittmann et al. 2010; Edmans, Gabaix 2011). This methodology incorporates the effect of the manager's preferences on the form of the optimal contract, but by construction it cannot study the effect of risk aversion independently from the effect of other preferences embedded in the utility function. In addition, the optimal contract derived with this type of model is very sensitive to the postulated form of the utility function (Chaigneau 2013). Third, a number of papers postulate mean-variance preferences, but they assume that compensation is linear in the performance measure(s), 
so that they do not derive implications for the effect of risk aversion on the form of the optimal contract (e.g., Zabojnik 1996; Jin 2002; Bolton et al. 2006). By contrast, this paper uses the mean-variance approach and studies the effect of risk aversion on the form of the optimal contract. It should be emphasized that this is not an optimal contracting paper, in the sense that we do not derive the optimal contract. Instead, our contribution is to present in a simple model a new reason for the sensitivity of pay to performance to be higher for low performances than for high performances.

Some papers have already recognized that punishments are more efficient than rewards to incentivize risk averse agents. Under certain conditions on the utility function and on the probability distribution of performances, Mirrlees (1975) shows that a contract based on extreme punishments approximates the first-best in a moral hazard problem with a risk averse agent. In a monitoring problem with a risk averse agent, Jewitt (1988) shows that the superiority of punishments over rewards is driven by properties of the utility function. Finally, other papers argue that convex contracts and protection against failure are more efficient: it is optimal to offer a convex, call-option like contract to a risk-neutral manager protected by limited liability (Innes 1990; Tirole 2006), which can be complemented with severance pay ( $\mathrm{Rau}, \mathrm{Xu} 2013$ ). Thus, the models of moral hazard with risk aversion on the one hand, and with risk neutrality and limited liability on the other hand, tend to generate different predictions regarding the structure of the optimal incentive contract. Punishments can be optimal with the former, while rewards are generally optimal with the latter. Whereas it is well-understood why rewards are optimal to motivate a risk neutral manager protected by limited liability, this paper contributes to explaining why punishments may be optimal to motivate a risk averse manager.

Section 1 presents the model. Section 2 derives the main results and discusses whether the pay-performance sensitivity is an appropriate measure of incentives. Section 3 considers step contracts. Section 4 extends the model to let effort affect both the expectation and the variance of the performance measure. The last Section presents conclusions.

\section{The contracting problem}

We consider a standard principal-agent relationship involving a manager and a principal which represents the firm's shareholders (think about the board). At time 0 , the manager exerts some costly effort $e \in[0, \infty)$. Effort shifts the mean of the contractible performance measure $\tilde{\pi}$, which is realized at time 1 , and which may represent either profits or the stock price, for instance. At time -1 , the risk-neutral principal offers to the manager a compensation contract $W(\pi)$, which specifies the manager's payment at time 1 as a function of $\pi$. For simplicity, the discount rate is zero.

We assume that the performance measure writes as:

$$
\tilde{\pi}=e+\tilde{\varepsilon} .
$$

The random variable $\tilde{\varepsilon}$ is realized at time 1 , but is unobservable. It is distributed according to the continuous probability density function (p.d.f.) $\varphi$, which is symmetric around zero, and the associated cumulative distribution function (c.d.f.) $\Phi$. The mean of $\tilde{\varepsilon}$ is normalized at zero and its variance is $\sigma^{2}$. Denote the p.d.f. of $\tilde{\pi}$ by $\psi$. 
In order to isolate the effect of risk aversion on contract design, we only consider the first and second-order moments of the distribution of payments received by the manager. Our reduced-form model assumes that the manager likes the mean and dislikes the variance of payments. In addition to being very tractable, this approach has the merit of focusing on risk aversion, to the exclusion of other factors. Formally, for any future random payment $\tilde{W}$ and effort $e$, the manager's objective function takes the form of a mean-variance criterion:

$$
E[\tilde{W} \mid e]-\omega \operatorname{var}[\tilde{W} \mid e]-\frac{c}{2} e^{2},
$$

where $\omega>0$ is the weight attributed to variance, and the third term if the cost of effort. The parameter $\omega$ is therefore a measure of risk aversion. The manager has nonnegative reservation utility $\bar{U}$, defined as the minimum value of (2) in equilibrium such that he accepts the contract $W(\pi)$. At time 0 , given a compensation contract $W(\pi)$, the manager chooses $e$ to maximize his objective function.

For any given $w$, the compensation contract $W(\pi)$ may be decomposed as $W(\pi)=w+f(\pi)$. We assume that the probability distribution is such that any optimal compensation profile is nondecreasing in the performance measure. The problem of the principal is to design $W(\pi)$ to minimize the expected cost of compensation $E[W(\tilde{\pi})]$, subject to the constraints that the manager accepts the contract (the participation constraint) and exerts a given level of effort $e^{*}$ (the incentive constraint).

A given contract $\{w, f\}$ satisfies the participation constraint if and only if:

$$
w+E\left[f(\tilde{\pi}) \mid e^{*}\right]-\omega \operatorname{var}\left[f(\tilde{\pi}) \mid e^{*}\right]-c \frac{e^{* 2}}{2} \geq \bar{U} .
$$

For a given $f$, define $\underline{w}$ as the value of $w$ which satisfies (3) as an equality. For (3) to be satisfied, we must have $w \geq \underline{w}$.

We assume that the effort cost $c$ is large enough for the first-order approach to be valid for all given compensation contracts under consideration (this imposes that we consider a bounded set of contracts). This is simply a sufficient condition for the effort choice problem to be always concave when required, even when the compensation profile is convex. This technical requirement guarantees an interior solution to the effort choice problem. For a given $f$, the necessary and sufficient first-order condition with respect to effort is then:

$$
E\left[f^{\prime}(\tilde{\pi}) \mid e\right]-\omega \frac{\partial}{\partial e} \operatorname{var}[f(\tilde{\pi}) \mid e]-c e=0 .
$$

The contract $f$ must be such that this equation is verified at $e=e^{*}$, so that the equilibrium level of effort is $e^{*}$. We call this condition the incentive constraint.

We define the first-best cost of eliciting effort $e^{*}, C^{F B}\left(e^{*}\right)$, and the first-best wage $w^{*}$, as:

$$
C^{F B}\left(e^{*}\right) \equiv \bar{U}+c \frac{e^{* 2}}{2} \equiv w^{*} .
$$

For a given $f$ which satisfies the incentive constraint in (4) for $e=e^{*}$ and a given $w \geq \underline{w}$ which satisfies the participation constraint in (3) for this $f$, the second-best cost of elicit- 
ing effort $e^{*}$ is:

$$
C^{S B}\left(e^{*}\right) \equiv E\left[W(\tilde{\pi}) \mid e^{*}\right]=w+E\left[f(\tilde{\pi}) \mid e^{*}\right] .
$$

Using (3) and the definition of $\underline{w}$ yields:

$$
C^{S B}\left(e^{*}\right)=\bar{U}+c \frac{e^{* 2}}{2}+\omega \operatorname{var}\left[f(\tilde{\pi}) \mid e^{*}\right]+(w-\underline{w}) .
$$

Given $f$ and $w$, we define the agency cost of eliciting effort $e^{*}$ as:

$$
A C_{f, w}\left(e^{*}\right) \equiv C^{S B}\left(e^{*}\right)-C^{F B}\left(e^{*}\right)=\omega \operatorname{var}\left[f(\tilde{\pi}) \mid e^{*}\right]+(w-\underline{w}) .
$$

Since $C^{F B}\left(e^{*}\right)$ is given, the principal's objective of minimizing the expected cost of compensation is equivalent to minimizing the agency cost $A C_{f, w}\left(e^{*}\right)$ of eliciting a given effort $e^{*}$. First, for any given $f$, this implies setting $w=\underline{w}$, given (6) and the fact that we must have $w \geq \underline{w}$ for the participation constraint (3) to be satisfied. For any given $f$ which satisfies the incentive constraint in (4) for $e=e^{*}$, the agency cost of eliciting effort $e^{*}$ at the optimum is therefore:

$$
A C_{f}\left(e^{*}\right)=\omega \operatorname{var}\left[f(\tilde{\pi}) \mid e^{*}\right] .
$$

Second, since the agency cost is proportional to the variance of $f$ at the optimum, the problem of the principal may be rewritten as:

$$
\min _{f} \operatorname{var}\left[f(\tilde{\pi}) \mid e^{*}\right] \quad \text { s.t. (4) with } e=e^{*} \text {. }
$$

We say that a given contract $f$ is dominated if and only if the agency cost of implementing $e^{*}$ can be reduced. In view of (7), a contract $f$ is dominated if and only if there exists another contract which also satisfies the incentive constraint (4) for $e=e^{*}$, but which is characterized by a lower variance.

\section{The main results}

In this setting, we are going to show that a set of compensation contracts is dominated and is therefore suboptimal. The first result below is proven in the Appendix.

Proposition 1: Any contract such that

is dominated.

$$
\frac{\partial}{\partial e} \operatorname{var}\left[W(\tilde{\pi}) \mid e^{*}\right]>0
$$

Any contract $f$ for which the variance of compensation at the equilibrium effort is locally strictly increasing in effort is suboptimal. Indeed, a symmetrical contract $h$ with the same average slope and the same variance as $f$ can be constructed. However, this new contract provides more incentives, since a marginal increase in effort at the equilibrium level of effort reduces the variance of compensation - whereas a marginal increase in effort has the opposite effect with the initial contract $f$. The new contract $h$, which necessarily provides excessive incentives, may therefore be flattened. This diminishes 
the equilibrium variance of pay, and therefore reduces agency costs. The approach is illustrated in Figure 1, where the initial contract $f$ is convex, the symmetrical contract $h$ is concave, and its flattened transformation $\frac{1}{\theta} h$, with $\theta>1$, is the dotted line. Finally, the fixed wage $w$ is adjusted in order to satisfy the participation constraint. With mean-variance preferences, this vertical translation of payments does not affect either incentives or the variance of pay.

For example, consider a manager compensated with stock options. Then the variability of pay of a manager who exerts effort will be higher than the variability of pay of a manager who does not. This is because a manager who exerts effort shifts the probability distribution toward performance measures associated with a positive pay-performance sensitivity (where the option is in-the-money), whereas a manager who does not exert effort shifts the probability distribution toward performance measures associated with a zero pay-performance sensitivity (where the option is out-of-the-money).

Interestingly, the average pay-performance sensitivity of the dominated contract $f$ (the convex contract on Fig. 1) is higher than the pay-performance sensitivity of the new contract $\frac{1}{\theta} h$ (the concave dotted line on Fig. 1) which induces the same effort. Indeed, we show in Proof 1 in the Appendix that, in equilibrium,

$$
E\left[f^{\prime}(\tilde{\pi}) \mid e^{*}\right]=E\left[h^{\prime}(\tilde{\pi}) \mid e^{*}\right] .
$$

So that, since $\theta>1$,

$$
E\left[f^{\prime}(\tilde{\pi}) \mid e^{*}\right]>E\left[\frac{1}{\theta} h^{\prime}(\tilde{\pi}) \mid e^{*}\right] .
$$

Even though both contracts elicit the same level of effort $e^{*}$, an empirical (ex-post) measure of the pay-performance sensitivity with type- $f$ contracts will be larger on average than with type- $h$ contracts. This implies that the level of incentives provided by

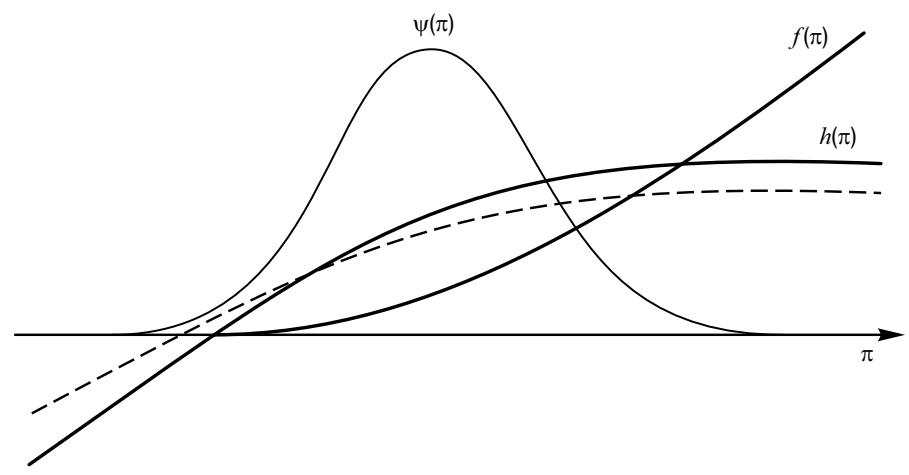

Fig. 1. Managerial compensation as a function of the measure of performance $\pi$

Notes: The convex contract $f$ is given. The concave contract $h$ is the transformation of the convex contract $f$ with the same average slope and the same variance described in the Proof of Proposition 1. The concave contract with a dotted line is the flattened version of the concave contract $h$ which induces the same level of effort as the initial convex contract. 
different contracts may only be ranked according to the pay-performance sensitivity criterion if these contracts all share the same form - or if $\omega=0$, so that the manager is risk neutral. This remark matters all the more that the managerial compensation literature has tended to measure incentives using the sensitivity of the manager's pay to his firm's performance (Jensen, Murphy 1990; Murphy 1999). More generally, this emphasizes that incentives can be strong even though the pay-performance sensitivity is low when measured ex-post. Intuitively, this will be the case when the incentive mechanism (strong punishments for poor performances, say) is rarely observed in equilibrium (under high effort).

Proposition 1 renders convex contracts suboptimal:

Proposition 2: It is suboptimal for $W(\pi)$ to be convex in $\pi$.

This is because the variance of compensation at the equilibrium level of effort is strictly increasing in effort with a convex contract, which is suboptimal. These results suggest that managers who are averse to the variance of their pay are more efficiently incentivized when the sensitivity of their pay to their performance is higher for low performances than for high performances.

Lastly, we argue that restricting attention to symmetric probability distributions needs not be without much loss of generality. Indeed, to the extent that a function of a random variable has a symmetric probability distribution, then we can apply the reasoning above to this new variable. We first make this point in the case of a random variable which follows a lognormal distribution. Consider a lognormally distributed variable $\tilde{\pi}$, and a compensation contract which writes as $W(\pi)=a+b \pi$, say. Clearly, it is equivalent to compensate the manager based on the normally distributed variable $\tilde{x} \equiv \ln (\tilde{\pi})$ and with the formula $W(x)=a+b \exp \{x\}$. These two contracts are equivalent, in the sense that they are associated with the same probability distribution of payments. It follows that if one is suboptimal, then the other is suboptimal as well. In this example, our model and our results apply to the contract $W(x)$, since the distribution of $\tilde{x}$ is symmetric. More generally, consider any random variable $\tilde{\pi}$, whose transformation $\tilde{x} \equiv f(\tilde{\pi})$ is symmetrically distributed. Suppose that we compensation contract writes as $W(\pi)=g(\pi)$. Then our model applies to the contract $W(x)=g\left(f^{-1}(x)\right)$, where the compensation of the manager is expressed as a function of the symmetrically distributed random variable $\tilde{x}$.

\section{Step contracts}

To take into account a lower bound on payments, potentially due to limited liability, we now study step contracts. We define a step contract as follows: it pays $m$ for $\pi<e^{*}+p$, and $M$ for $\pi \geq e^{*}+p$, with $M>m$ and a given $p$. If a step contract is characterized by $p<0$, then we say that it uses "sticks". Indeed, with such a contract, the absolute value of $m-E[W(\tilde{\pi})]$ is larger than that of $M-E[W(\tilde{\pi})]$, by construction ${ }^{4}$. Otherwise we say that the contract uses "carrots".

\footnotetext{
${ }^{4}$ We prove this statement in the proof of Proposition 3.
} 
Suppose that payments are bounded from below, in the sense that $W(\pi) \geq L$ for any $\pi$ (setting $L=0$ makes this a limited liability constraint). The next result shows that this "lower bound constraint" is always binding at the optimum.

Proposition 3: The optimal step contract is characterized by $m=L$.

For step contracts which induce the same given level of effort and for which the participation constraint binds, we show in the proof of Proposition 3 that both $d \Phi(p) / d m$ and $d M / d m$ are strictly positive for any value of $m$ : both $p$ and $M$ are strictly increasing in $m$ at the margin. This in turn implies that the parameters $p$ and $M$ of contracts which satisfy both the participation constraint as an equality and the incentive constraint are strictly increasing in $m$.

Intuitively, equating the low payment $m$ to the lower bound $L$ enables to set the threshold $p$ at the lowest possible level. This maximizes the difference between the variability of pay conditional on the equilibrium effort $e^{*}$ and a lower level of effort. In turn, this increases the effort incentives of a risk averse manager, and it also minimizes the variance of pay conditional on $e^{*}$ in equilibrium, i.e., the agency cost.

Assume that $c, e^{*}$, and $\omega$ are bounded away from zero and $c e^{*}$ is bounded from above. Then, in the limit case where $L$ tends to minus infinity, the optimal contract is essentially the Mirrlees (1975) mechanism, and the second-best outcome approximates the first-best:

Proposition 4: If $L \rightarrow-\infty$, then $p \rightarrow-\infty, M \rightarrow w^{*}$, and the agency cost is approximately zero.

These results suggest that the absence of extreme punishments in observed contracts may not be due to their economic inefficiency but to constraints on contracting.

\section{Extension: multiplicative structure}

This section extends the model with a more general structure for the performance measure, in the spirit of Edmans et al. (2009) ${ }^{5}$. We establish in which case convex contracts are still suboptimal when effort affects not only the mean but also the variance of the performance measure.

We now assume that the performance measure writes as:

$$
\tilde{\pi}=e+\tilde{\varepsilon}+\gamma e \tilde{\varepsilon},
$$

where $\gamma \in\left(-1 / e^{*}, \infty\right)$. With $\gamma=0$, the performance measure is additive in effort and noise (this corresponds to the baseline model). With $\gamma \neq 0$, there is an additional multiplicative term. As before, the expectation of the performance measure is increasing in effort. In addition, with $\gamma>0$, the variance of the performance measure is now increasing in effort: $d \operatorname{var}(\tilde{\pi}) / d e>0$, where:

$$
\frac{d \operatorname{var}(\tilde{\pi})}{d e}=\frac{d \operatorname{var}((1+\gamma e) \tilde{\varepsilon})}{d e}=2 \gamma(1+\gamma e) \sigma^{2} .
$$

\footnotetext{
${ }^{5}$ Using the notations of our paper, their specification for the performance measure is essentially $1+e+\tilde{\varepsilon}+e \tilde{\varepsilon}$.
} 
On the contrary, with $\gamma \in\left(-1 / e^{*}, 0\right)$, we have $d \operatorname{var}(\tilde{\pi}) / d e<0$.

Under this new structure, Proposition 2 still holds if the variance of the performance measure is decreasing in effort:

Corollary 1: With a performance measure as in (9), if $\gamma \in\left(-1 / e^{*}, 0\right)$, then it is suboptimal for $W(\pi)$ to be convex in $\pi$.

With a performance measure as in (9), the expected pay and the variance of pay will be the same under any contract as with $\gamma=0$. As above, a concave contract provides more incentives because a marginal increase in effort diminishes the variance of pay more than with a convex contract ${ }^{6}$. With $\gamma \in\left(-1 / e^{*}, 0\right)$, a concave contract provides more effort incentives for an additional reason: it concentrates the slope on the downside, where effort has a stronger effect on the performance measure. Note that this additional effect is unrelated to the manager's risk aversion. As above, it is then possible to flatten the newly constructed concave contract so as to induce the same effort as the original convex contract for a lower variance of payment, i.e., for a lower agency cost.

However, with $\gamma>0$, the additional incentive effect goes in the other direction, so that it is in general not clear that convex contracts are dominated. In the limit case where the manager is approximately risk neutral $(\omega \rightarrow 0)$, the additional incentive effect dominates the former, and concave contracts are dominated:

Proposition 5: With a performance measure as in (9), if $\gamma>0$ and $\omega \rightarrow 0$, then it is suboptimal for $W(\pi)$ to be concave in $\pi$.

This result emphasizes that it is important to know the effect of effort on the variance of the performance measure to determine the form of the optimal contract. In particular, convex contracts provide more incentives to risk neutral managers if and only if the variance of the performance measure is increasing in effort (i.e., $\gamma>0$ ).

\section{Conclusions}

This paper studied the implications of an aversion towards the variance of payments for the design of managerial incentive contracts. In a simple principal-agent model in which effort affects the mean of the performance measure, we find that more effort incentives are provided by contracts which are such that the variance of pay decreases in effort. An important implication is that convex contracts, including stock options, are suboptimal in this setting.

The model can explain the paucity of bonus-type incentive schemes which reward good performances in most firms, and it can shed light on the decline in the relative importance of stock options for CEO compensation since 2000. The model can also contribute to explain the existence of incentive contracts with punishments for low performances such as dismissals, capped "bonuses", and inside debt. Indeed, these incentive schemes

\footnotetext{
${ }^{6}$ More precisely, a marginal increase in effort now also increases of $\tilde{\pi}$ at the margin, but $\operatorname{var}(f(\tilde{\pi}))=\operatorname{var}(h(\tilde{\pi}))$ by construction, so that the marginal increase in variance is the same under both contracts.
} 
are such that the variability of pay is low as long as performance is above a threshold. The model delivers two important practical implications. First, incentive schemes should generally be designed by taking into account the principle that the sensitivity of pay to performance should be decreasing in performance, i.e., they should rely more on "sticks" than on "carrots". Second, for such incentive schemes, pay-performance sensitivities as measured in equilibrium will be relatively low. This is because the performances of managers who exert high effort will tend to be good, so that punishments are rarely administered ex-post, even though their existence provides adequate incentives ex-ante.

One limitation of this paper is that it only considered the implications of risk aversion for the structure of the optimal contract. Future theoretical work could determine the implications of the preferences for higher-order moments of the distribution of wealth, notably the preference for a positive skewness and the aversion to kurtosis. In addition, future applied work could design mechanisms which take into account the principle established in this paper. This would be especially relevant for the design of dismissals as an incentive mechanism (e.g., Kaplan, Minton 2012), to filter out exogenous shocks to performance (e.g., Dittmann et al. 2013), for the regulation of CEO compensation (e.g., Dicks 2012; Chaigneau 2013), but also in other areas where the provision of incentives matters. In particular, risk aversion could be proxied by managerial characteristics such as wealth (Becker 2006) but also other attributes (Graham et al. 2013).

\section{References}

Bebchuk, L. A.; Fried, J. M. 2003. Executive compensation as an agency problem, Journal of Economic Perspectives 17(3): 71-92. http://dx.doi.org/10.1257/089533003769204362

Becker, B. 2006. Wealth and executive compensation, Journal of Finance 61(1): 379-397.

http://dx.doi.org/10.1111/j.1540-6261.2006.00839.x

Bolton, P.; Dewatripont, M. 2005. Contract theory. Cambridge, MA: MIT Press.

Bolton, P.; Scheinkman, J.; Xiong, W. 2006. Executive compensation and short-termist behaviour in speculative markets, Review of Economic Studies 73(3): 577-610.

http://dx.doi.org/10.1111/j.1467-937X.2006.00388.x

Chaigneau, P. 2013. Explaining the structure of CEO incentive pay with decreasing relative risk aversion, Journal of Economics and Business 67: 4-23.

http://dx.doi.org/10.1016/j.jeconbus.2012.10.002

Chaigneau, P. 2013. Risk-shifting and the regulation of bank CEOs' compensation, Journal of Financial Stability 9(4): 778-789. http://dx.doi.org/10.1016/j.jfs.2012.08.003

Coles, J. L.; Daniel, N. D.; Naveen, L. 2006. Managerial incentives and risk taking, Journal of Financial Economics 79(2): 431-468. http://dx.doi.org/10.1016/j.jfineco.2004.09.004

Dicks, D. 2012. Executive compensation and the role for corporate governance regulation, Review of Financial Studies 25(6): 1971-2004. http://dx.doi.org/10.1093/rfs/hhs055

Dittmann, I.; Maug, E. 2007. Lower salaries and no options? On the optimal structure of executive pay, Journal of Finance 62(1): 303-343. http://dx.doi.org/10.1111/j.1540-6261.2007.01208.x

Dittmann, I.; Maug, E.; Spalt, O. 2010. Sticks or carrots? Optimal CEO compensation when managers are loss averse, Journal of Finance 65(6): 2015-2050.

http://dx.doi.org/10.1111/j.1540-6261.2010.01609.x 
Dittmann, I.; Maug, E.; Spalt, O. 2011. Restricting CEO pay, Journal of Corporate Finance 17(4): 1200-1220. http://dx.doi.org/10.1016/j.jcorpfin.2011.04.007

Dittmann, I.; Maug, E.; Spalt, O. 2013. Indexing executive compensation contracts, Review of Financial Studies 26(12): 3182-3224. http://dx.doi.org/10.1093/rfs/hht052

Edmans, A.; Gabaix, X. 2009. Is CEO pay really inefficient? A survey of new optimal contracting theories, European Financial Management 15(3): 486-496.

http://dx.doi.org/10.1111/j.1468-036X.2009.00500.x

Edmans, A.; Gabaix, X. 2011. Tractability in incentive contracting, Review of Financial Studies 24(9): 2865-2894. http://dx.doi.org/10.1093/rfs/hhr044

Edmans, A.; Gabaix, X.; Landier, A. 2009. A multiplicative model of optimal CEO incentives in market equilibrium, Review of Financial Studies 22(12): 4881-4917.

http://dx.doi.org/10.1093/rfs/hhn117

Edmans, A.; Liu, Q. 2011. Inside debt, Review of Finance 15(1): 75-102.

http://dx.doi.org/10.1093/rof/rfq008

Francis, B.; Hasan, I.; Sharma, Z. 2011. Incentives and innovation: evidence from CEO compensation contracts, Working paper. Bank of Finland.

Garen, J. E. 1994. Executive compensation and principal-agent theory, Journal of Political Economy 102(6): 1175-1199. http://dx.doi.org/10.1086/261967

Gibbons, R.; Murphy, K. J. 1992. Optimal incentive contracts in the presence of career concerns: theory and evidence, Journal of Political Economy 100(3): 468-505.

http://dx.doi.org/10.1086/261826

Graham, J. R.; Harvey, C. R.; Puri, M. 2013. Managerial attitudes and corporate actions, Journal of Financial Economics 109(1): 103-121. http://dx.doi.org/10.1016/j.jfineco.2013.01.010

Hall, B. J.; Murphy, K. J. 2002. Stock options for undiversified executives, Journal of Accounting and Economics 33(1): 3-42. http://dx.doi.org/10.1016/S0165-4101(01)00050-7

Hall, B. J.; Murphy, K. J. 2003. The trouble with stock options, Journal of Economic Perspectives 17(3): 49-70. http://dx.doi.org/10.1257/089533003769204353

Hemmer, T.; Kim, O.; Verrecchia, R. E. 2000. Introducing convexity into optimal compensation contracts, Journal of Accounting and Economics 28(3): 307-327.

http://dx.doi.org/10.1016/S0165-4101(00)00008-2

Holmstrom, B. 1979. Moral hazard and observability, Bell Journal of Economics 10(1): 74-91. http://dx.doi.org/10.2307/3003320

Holmstrom, B.; Tirole, J. 1993. Market liquidity and performance monitoring, Journal of Political Economy 101(4): 678-709. http://dx.doi.org/10.1086/261893

Innes, R. D. 1990. Limited liability and incentive contracting with ex-ante action choices, Journal of Economic Theory 52(1): 45-67. http://dx.doi.org/10.1016/0022-0531(90)90066-S

Jensen, M. C.; Murphy, K. J. 1990. Performance pay and top-management incentives, Journal of Political Economy 98(2): 225-64. http://dx.doi.org/10.1086/261677

Jewitt, I. 1988. Justifying the first-order approach to principal-agent problems, Econometrica 56(5): 1177-1190. http://dx.doi.org/10.2307/1911363

Jin, L. 2002. CEO compensation, diversification, and incentives, Journal of Financial Economics 66(1): 29-63. http://dx.doi.org/10.1016/S0304-405X(02)00150-2

Johnstone, D. J.; Lindley, D. V. 2011. Elementary proof that mean-variance implies quadratic utility, Theory and Decision 70(2): 149-155. http://dx.doi.org/10.1007/s11238-010-9194-7

Kaplan, S.; Minton, B. 2012. How has CEO turnover changed? International Review of Finance 12(1): 57-87. http://dx.doi.org/10.1111/j.1468-2443.2011.01135.x 
Laffont, J.-J.; Martimort, D. 2002. The theory of incentives. Princeton, NJ: Princeton University Press.

Mirrlees, J. A. 1975. The theory of moral hazard and unobservable behavior, Part 1. Mimeo. Oxford.

Manso, G. 2011. Motivating innovation, Journal of Finance 66(5): 1823-1860.

http://dx.doi.org/10.1111/j.1540-6261.2011.01688.x

Murphy, K. J. 1999. Executive compensation, in O. Ashenfelter, D. E. Card (Eds.). Handbook of labor economics New York: North Holland, 2485-2563.

http://dx.doi.org/10.1016/S1573-4463(99)30024-9

Rau, P. R.; Xu, J. 2013. How do ex ante severance pay contracts fit into optimal executive incentive schemes?, Journal of Accounting Research 51(3): 631-671.

http://dx.doi.org/10.1111/joar.12001

Salanié, B. 1997. The economics of contracts. Cambridge, MA: MIT Press.

Sundaram, R. K.; Yermack, D. L. 2007. Pay me later: inside debt and its role in managerial compensation, Journal of Finance 62(4): 1551-1588.

http://dx.doi.org/10.1111/j.1540-6261.2007.01251.x

Tirole, J. 2006. The theory of corporate finance. Princeton, NJ: Princeton University Press.

Zabojnik, J. 1996. Pay-performance sensitivity and production uncertainty, Economic Letters 53(3): 291-296. http://dx.doi.org/10.1016/S0165-1765(96)00938-X

\section{APPENDIX}

\section{Proof of Proposition 1:}

For any contract such that $\frac{\partial}{\partial e} \operatorname{var}\left[W(\tilde{\pi}) \mid e^{*}\right]>0$, i.e. such that $\frac{\partial}{\partial e} \operatorname{var}\left[f(\tilde{\pi}) \mid e^{*}\right]>0$, we need to show that it is possible to design another contract that induces the same effort $e^{*}$ at a lower variance. Indeed, we know from section 1 that, given the new contract, $w$ adjusts to satisfy the participation constraint: $w=\underline{w}$, where $\underline{w}$ satisfies the participation constraint (3) as an equality (note that a change in $w$ neither affects the incentive constraint in (4) nor the variance of compensation). Finally, we know from (7) that the new contract with a lower variance is associated with a lower agency cost, and therefore dominates the former.

To this end, in the $(\pi, W)$ space, consider the contract symmetrical to $f$ with respect to the point $\left(e^{*}, f\left(e^{*}\right)\right)$ : the function defining the new compensation profile is obtained by taking a function symmetrical to $f$ with respect to the horizontal line going through the point $\left(e^{*}, f\left(e^{*}\right)\right)$, then taking a function symmetrical to this new function with respect to the vertical line going through the same point. Denote the function thus obtained by $h$. To start with, assume that effort does not change. Then, we show in Proof 1 later in the Appendix that:

$$
\begin{gathered}
\operatorname{var}\left[h(\tilde{\pi}) \mid e^{*}\right]=\operatorname{var}\left[f(\tilde{\pi}) \mid e^{*}\right], \\
E\left[h^{\prime}(\tilde{\pi}) \mid e^{*}\right]=E\left[f^{\prime}(\tilde{\pi}) \mid e^{*}\right], \\
\frac{\partial}{\partial e} \operatorname{var}\left[h(\tilde{\pi}) \mid e^{*}\right]<0 .
\end{gathered}
$$


However, effort, as defined in (4) by the first-order condition of the manager's problem, will increase under $h$. Indeed, $e^{*}$ solves:

$$
e^{*}=\frac{1}{c}\left(E\left[f^{\prime}(\tilde{\pi}) \mid e^{*}\right]-\omega \frac{\partial}{\partial e} \operatorname{var}\left[f(\tilde{\pi}) \mid e^{*}\right]\right),
$$

with $\frac{\partial}{\partial e} \operatorname{var}\left[f(\tilde{\pi}) \mid e^{*}\right]>0$. The effort $\hat{e}$ induced by $h$ solves:

$$
\hat{e}=\frac{1}{c}\left(E\left[h^{\prime}(\tilde{\pi}) \mid \hat{e}\right]-\omega \frac{\partial}{\partial e} \operatorname{var}[h(\tilde{\pi}) \mid \hat{e}]\right) .
$$

But, since $E\left[h^{\prime}(\tilde{\pi}) \mid \hat{e}\right]=E\left[f^{\prime}(\tilde{\pi}) \mid e^{*}\right]$ and $\frac{\partial}{\partial e} \operatorname{var}[h(\tilde{\pi}) \mid \hat{e}]<0$, we have $\hat{e}>e^{*}$.

For any $e$ and any $\theta>0$ :

$$
\begin{gathered}
E\left[\frac{1}{\theta} h^{\prime}(\tilde{\pi}) \mid e\right]=\frac{1}{\theta} E\left[h^{\prime}(\tilde{\pi}) \mid e\right], \\
\frac{\partial}{\partial e} \operatorname{var}\left[\frac{1}{\theta} h(\tilde{\pi}) \mid e\right]=\frac{1}{\theta^{2}} \frac{\partial}{\partial e} \operatorname{var}[h(\tilde{\pi}) \mid e] .
\end{gathered}
$$

It follows from these two equations that the terms $E\left[\frac{1}{\theta} h^{\prime}(\tilde{\pi}) \mid e\right]$ and $\frac{\partial}{\partial e} \operatorname{var}\left[\frac{1}{\theta} h(\tilde{\pi}) \mid e\right]$ are monotonically decreasing in $\theta$. Furthermore, their first-order derivatives with respect to $\theta$ exist and are finite, for $\theta \in(0, \infty)$. Lastly, for $\theta=1$, effort $\hat{e}$ is induced. Therefore, there exists a $\theta>1$ such that:

$$
e^{*}=\frac{1}{c}\left(E\left[\frac{1}{\theta} h^{\prime}(\tilde{\pi}) \mid e^{*}\right]-\omega \frac{\partial}{\partial e} \operatorname{var}\left[\frac{1}{\theta} h(\tilde{\pi}) \mid e^{*}\right]\right),
$$

where $e^{*}$ solves (11).

The compensation profile $\frac{1}{\theta} h$ induces the same effort as $f$, but at a lower agency cost: the variance of $h$ is equal to the variance of $f$, so that the variance of $\frac{1}{\theta} h$ is lower than the variance of $f$. The proof is complete.

\section{Proof 1:}

Consider the function $g$, symmetrical to $f$ with respect to the horizontal line going through the point $\left(e^{*}, f\left(e^{*}\right)\right)$. Up to an additive constant equal to $2 f\left(e^{*}\right), g$ is the opposite of $f: g(\pi)=-f(\pi)+2 f\left(e^{*}\right)$. Its variance writes as:

$$
\begin{aligned}
& \operatorname{var}[g(\tilde{\pi})] \equiv E\left[(g(\tilde{\pi})-E[g(\tilde{\pi})])^{2}\right]=E\left[\left(-f(\tilde{\pi})+2 f\left(e^{*}\right)+E[f(\tilde{\pi})]-2 f\left(e^{*}\right)\right)^{2}\right]= \\
& E\left[(f(\tilde{\pi})-E[f(\tilde{\pi})])^{2}\right] \equiv \operatorname{var}[f(\tilde{\pi})] .
\end{aligned}
$$

Consider the function $h$, symmetrical to $g$ with respect to the vertical line going through the point $\left(e^{*}, f\left(e^{*}\right)\right)$. By definition, it writes as:

$$
h(\pi)=g\left(\pi-2\left(\pi-e^{*}\right)\right)=g\left(-\pi+2 e^{*}\right),
$$

where $\varphi(x)$ is the p.d.f. for a symmetrically distributed variable $x$ of mean 0 and variance $\rho^{2}$. Because the p.d.f. of $\tilde{\pi}$ is centred around the mean $e^{*}$ of $\tilde{\pi}$, we have $\psi\left(-\pi+2 e^{*}\right)=\psi(\pi)$. The expectation of $h(\tilde{\pi})$ is: 


$$
\begin{aligned}
& E[h(\tilde{\pi})] \equiv \int_{-\infty}^{\infty} h(\pi) \psi(\pi) d \pi=\int_{-\infty}^{\infty} g\left(-\pi+2 e^{*}\right) \psi(\pi) d \pi= \\
& \int_{-\infty}^{\infty} g(\pi) \psi\left(-\pi+2 e^{*}\right) d \pi=\int_{-\infty}^{\infty} g(\pi) \psi(\pi) d \pi \equiv E[g(\tilde{\pi})],
\end{aligned}
$$

where the second equality uses the definition of $g$, the third involves a change of variable, and the fourth uses the symmetry of $\varphi$. Equalities below involve the same steps, plus the fact that $E[g(\tilde{\pi})]$ is a constant:

$$
\begin{aligned}
& \operatorname{var}[h(\tilde{\pi})] \equiv \int_{-\infty}^{\infty}(h(\pi)-E[h(\tilde{\pi})])^{2} \psi(\pi) d \pi=\int_{-\infty}^{\infty}\left(g\left(-\pi+2 e^{*}\right)-E[g(\tilde{\pi})]\right)^{2} \psi(\pi) d \pi= \\
& \int_{-\infty}^{\infty}(g(\pi)-E[g(\tilde{\pi})])^{2} \psi\left(-\pi+2 e^{*}\right) d \pi=\int_{-\infty}^{\infty}(g(\pi)-E[g(\tilde{\pi})])^{2} \psi(\pi) d \pi \equiv \operatorname{var}[g(\tilde{\pi})] .
\end{aligned}
$$

Eventually, combining (16) with (19):

$$
\operatorname{var}[h(\tilde{\pi})]=\operatorname{var}[g(\tilde{\pi})]=\operatorname{var}[f(\tilde{\pi})] .
$$

The second part of the proof compares $E\left[h^{\prime}(\tilde{\pi})\right]$ to $E\left[f^{\prime}(\tilde{\pi})\right]$. Using the definition of $g$ and remembering that $f\left(e^{*}\right)$ is a constant, we get $g^{\prime}(\pi)=-f^{\prime}(\pi)$, and $E\left[g^{\prime}(\tilde{\pi})\right]=-E\left[f^{\prime}(\tilde{\pi})\right]$. Besides, the derivative of $h$ with respect to $\pi$ is $h^{\prime}(\pi)=-g^{\prime}\left(-\pi+2 e^{*}\right)$. Going once again through the same steps:

$$
\begin{aligned}
& E\left[h^{\prime}(\tilde{\pi})\right] \equiv \int_{-\infty}^{\infty} h^{\prime}(\pi) \psi(\pi) d \pi=\int_{-\infty}^{\infty}-g^{\prime}\left(-\pi+2 e^{*}\right) \psi(\pi) d \pi= \\
& \int_{-\infty}^{\infty}-g^{\prime}(\pi) \psi\left(-\pi+2 e^{*}\right) d \pi=\int_{-\infty}^{\infty}-g^{\prime}(\pi) \psi(\pi) d \pi \equiv-E\left[g^{\prime}(\tilde{\pi})\right] .
\end{aligned}
$$

Combining these two results, $E\left[h^{\prime}(\tilde{\pi})\right]=-E\left[g^{\prime}(\tilde{\pi})\right]=E\left[f^{\prime}(\tilde{\pi})\right]$.

The third part of the proof shows that the derivatives with respect to effort of the conditional variances of $f(\tilde{\pi})$ and $h(\tilde{\pi})$ have opposite signs. On the one hand, using the definition of $g$ :

$$
\frac{\partial}{\partial e} \operatorname{var}[g(\tilde{\pi}) \mid e]=\frac{\partial}{\partial e} \operatorname{var}\left[-f(\tilde{\pi})+2 f\left(e^{*}\right) \mid e\right]=\frac{\partial}{\partial e} \operatorname{var}[-f(\tilde{\pi}) \mid e]=\frac{\partial}{\partial e} \operatorname{var}[f(\tilde{\pi}) \mid e] .
$$

On the other hand,

$$
\begin{aligned}
& \frac{\partial}{\partial e} \operatorname{var}[g(\tilde{\pi}) \mid e]=\frac{\partial}{\partial e} \int_{-\infty}^{\infty}(g(\pi)-E[g(\tilde{\pi})])^{2} \varphi(\varepsilon) d \varepsilon= \\
& \int_{-\infty}^{\infty} 2\left[\frac{\partial}{\partial e} g(\pi)-\frac{\partial}{\partial e} E[g(\tilde{\pi})]\right](g(\pi)-E[g(\tilde{\pi})]) \varphi(\varepsilon) d \varepsilon .
\end{aligned}
$$

In addition,

$$
\begin{aligned}
& \frac{\partial}{\partial e} \operatorname{var}[h(\tilde{\pi})]=\frac{\partial}{\partial e} \int_{-\infty}^{\infty}(h(\pi)-E[h(\tilde{\pi})])^{2} \varphi(\varepsilon) d \varepsilon= \\
& \int_{-\infty}^{\infty}-2\left[\frac{\partial}{\partial e} g\left(-\pi+2 e^{*}\right)-\frac{\partial}{\partial e} E\left[g\left(-\tilde{\pi}+2 e^{*}\right)\right]\right]\left(g\left(-\pi+2 e^{*}\right)-E\left[g\left(-\tilde{\pi}+2 e^{*}\right)\right]\right) \varphi(\varepsilon) d \varepsilon= \\
& \int_{-\infty}^{\infty}-2\left[\frac{\partial}{\partial e} g(\pi)-\frac{\partial}{\partial e} E[g(\tilde{\pi})]\right](g(\pi)-E[g(\tilde{\pi})]) \varphi(-\varepsilon) d \varepsilon=
\end{aligned}
$$




$$
\int_{-\infty}^{\infty}-2\left[\frac{\partial}{\partial e} g(\pi)-\frac{\partial}{\partial e} E[g(\tilde{\pi})]\right](g(\pi)-E[g(\tilde{\pi})]) \varphi(\varepsilon) d \varepsilon=-\frac{\partial}{\partial e} \operatorname{var}[g(\tilde{\pi}) \mid e],
$$

where the second equality uses the definition of $h$ in (17), the third equality involves a change of variable $(\varepsilon=-\varepsilon)$, the fourd equality uses the symmetry of $\varphi$ around the origin (which implies $\varphi(-\varepsilon)=\varphi(\varepsilon)$ ), and the fifth equality equates with (22). Combining (21) with (23), we have $\frac{\partial}{\partial e} \operatorname{var}[h(\tilde{\pi}) \mid e]=-\frac{\partial}{\partial e} \operatorname{var}[f(\tilde{\pi}) \mid e]$ for every $e$.

\section{Proof of Proposition 2:} It is sufficient to show that if $f$ is convex in $\pi$, then $\frac{\partial}{\partial e} \operatorname{var}\left[f(\tilde{\pi}) \mid e^{*}\right]>0$. Proposition
1 then gives us the desired result.

To start with,

$$
\begin{aligned}
& \frac{\partial}{\partial e} \operatorname{var}\left[f(\tilde{\pi}) \mid e^{*}\right]=\int_{-\infty}^{\infty} \frac{\partial}{\partial e}\left(f\left(e^{*}+\varepsilon\right)-E\left[f\left(e^{*}+\tilde{\varepsilon}\right)\right]\right)^{2} \varphi(\varepsilon) d \varepsilon= \\
& \int_{-\infty}^{\infty} 2(f(\pi)-E[f(\tilde{\pi})])\left(f^{\prime}(\pi)-E\left[f^{\prime}(\tilde{\pi})\right]\right) \varphi(\varepsilon) d \varepsilon .
\end{aligned}
$$

The function $f$ being increasing and convex in the performance measure:

Therefore,

$$
\begin{gathered}
\frac{\partial f(\pi)}{\partial \varepsilon}=\frac{\partial f(\pi)}{\partial \pi} \frac{\partial \pi}{\partial \varepsilon}=f^{\prime}(\pi)>0, \\
\frac{\partial f^{\prime}(\pi)}{\partial \varepsilon}=\frac{\partial f^{\prime}(\pi)}{\partial \pi} \frac{\partial \pi}{\partial \varepsilon}=f^{\prime \prime}(\pi)>0 .
\end{gathered}
$$

Additionally,

$$
\operatorname{cov}\left(f(\tilde{\pi}), f^{\prime}(\tilde{\pi})\right)>0 .
$$

$$
\operatorname{cov}\left(f(\tilde{\pi}), f^{\prime}(\tilde{\pi})\right)=\int_{-\infty}^{\infty}(f(\pi)-E[f(\tilde{\pi})])\left(f^{\prime}(\pi)-E\left[f^{\prime}(\tilde{\pi})\right]\right) \varphi(\varepsilon) d \varepsilon .
$$

Which is positive because of (25). Applying these two results to (24) completes the proof.

\section{Proof of Proposition 3:}

A step contract is defined by its floor $m$, its cap $M$, and the cutoff $p$. Bearing in mind that:

$$
\begin{gathered}
E[W(\tilde{\pi})]=m \Phi(p)+M(1-\Phi(p)), \\
\operatorname{var}[W(\tilde{\pi})]=(m-E[W(\tilde{\pi})])^{2} \Phi(p)+(M-E[W(\tilde{\pi})])^{2}(1-\Phi(p)),
\end{gathered}
$$

we compute derivatives with step contracts:

$$
\begin{aligned}
& E\left[W^{\prime}(\tilde{\pi})\right]=\lim _{a \rightarrow 0} \int_{p-a}^{p+a} \frac{M-m}{2 a} d \Phi(\varepsilon)=(M-m) \lim _{a \rightarrow 0} \frac{1}{2 a} \int_{p-a}^{p+a} d \Phi(\varepsilon)= \\
& (M-m) \lim _{a \rightarrow 0} \frac{\Phi(p+a)-\Phi(p-a)}{2 a}=(M-m) \varphi(p) .
\end{aligned}
$$




$$
\begin{aligned}
& \frac{\partial}{\partial e} \operatorname{var}[W(\tilde{\pi})]=\frac{\partial}{\partial e}\left\{\int_{-\infty}^{p-e+e^{*}}(m-E[W(\tilde{\pi})])^{2} d \Phi(\varepsilon)+\int_{p-e+e^{*}}^{\infty}(M-E[W(\tilde{\pi})])^{2} d \Phi(\varepsilon)\right\}= \\
& -\varphi(p)(m-E[W(\tilde{\pi})])^{2}+\varphi(p)(M-E[W(\tilde{\pi})])^{2} .
\end{aligned}
$$

This expression is negative if and only if

$$
|m-E[W(\tilde{\pi})]|>|M-E[W(\tilde{\pi})]|,
$$

which is the case if and only if $p$ is lower than zero (because of (26) and the fact that, for a symmetrically distributed random variable, $\Phi(0)=0.5)$.

The incentive constraint is:

$$
(M-m) \varphi(p)-\omega \varphi(p)\left[M^{2}-m^{2}-2(M-m) E[W(\tilde{\pi})]\right]=c e^{*},
$$

or

$$
(M-m)[\varphi(p)-\omega \varphi(p)(M+m-2 m \Phi(p)-2 M(1-\Phi(p)))]=c e^{*} .
$$

We rewrite the incentive constraint in (27) as:

$$
\eta(m, M(m)) \equiv c e^{*} .
$$

Suppose that a given step contract satisfies the incentive constraint. Following a change in $m$, the adjustment in $M$ required for the incentive constraint to be satisfied, holding $p$ constant, is:

$$
\frac{d M}{d m}=-\frac{\frac{\partial}{\partial m} \eta(m, M(m))}{\frac{\partial}{\partial M} \eta(m, M(m))} .
$$

The participation constraint is:

$$
m \Phi(p)+M(1-\Phi(p))-\omega\left[(m-E[W(\tilde{\pi})])^{2} \Phi(p)+(M-E[W(\tilde{\pi})])^{2}(1-\Phi(p))\right]=\bar{U}+c \frac{e^{*}}{2}
$$

We rewrite (29) as:

$$
\mu(m,[\Phi(p)](m)) \equiv \bar{U}+c \frac{e^{*}}{2} .
$$

Suppose that a given step contract satisfies the participation constraint. Following a change in $m$, the adjustment in $p$ required for the participation constraint to be satisfied, holding $M$ constant, is:

$$
\frac{d \Phi(p)}{d m}=-\frac{\frac{\partial}{\partial m} \mu(m,[\Phi(p)](m))}{\frac{\partial}{\partial p} \mu(m,[\Phi(p)](m))}
$$

A given contract which satisfies both the participation constraint and the incentive constraint is dominated if marginally changing $m$, and adjusting $M$ and $p$ accordingly to satisfy the incentive and participation constraints (so that the manager still participates and still selects the same level of effort $e^{*}$ ), diminishes the expected payments made to the manager (the second-best cost of eliciting effort $e^{*}$ ). We now calculate the sign of 
the total derivative of expected payments to the manager with respect to $m$ :

$$
\begin{aligned}
& \frac{d}{d m} E[W(\tilde{\pi})]=\Phi(p)+m \frac{d \Phi(p)}{d m}+\frac{d M}{d m}(1-\Phi(p))-M \frac{d \Phi(p)}{d m}= \\
& \Phi(p)+(M-m)\left(-\frac{d \Phi(p)}{d m}\right)+\frac{d M}{d m}(1-\Phi(p)) .
\end{aligned}
$$

Start with the $\frac{d \Phi(p)}{d m}$ term. On the one hand,

$$
\begin{aligned}
& \frac{\partial}{\partial m} \mu(m,[\Phi(p)](m))=\Phi(p)-\omega[2(1+\Phi(p))(m-E[W(\tilde{\pi})]) \Phi(p)+ \\
& 2(1+\Phi(p))(M-E[W(\tilde{\pi})])(1-\Phi(p))]=\Phi(p)-2 \omega(1+\Phi(p))[m \Phi(p)- \\
& m(\Phi(p))^{2}-M(1-\Phi(p)) \Phi(p)+M(1-\Phi(p))-m \Phi(p)(1-\Phi(p))- \\
& \left.M(1-\Phi(p))^{2}\right]=\Phi(p) .
\end{aligned}
$$

On the other hand,

$$
\begin{aligned}
& \frac{\partial}{\partial \Phi(p)} \mu(m,[\Phi(p)](m))=m-M-2 \omega[(M-m)(m-E[W(\tilde{\pi})]) \Phi(p)+ \\
& (m-m \Phi(p)-M(1-\Phi(p)))^{2}+(M-m)(M-E[W(\tilde{\pi})])(1-\Phi(p))- \\
& \left.(m-m \Phi(p)-M(1-\Phi(p)))^{2}\right]=(M-m)[-1-2 \omega[m \Phi(p)+M(1-\Phi(p))- \\
& E[W(\tilde{\pi})]]]=-(M-m) .
\end{aligned}
$$

So that

Next, consider the $\frac{d M}{d m}$ term. On the one hand,

$$
\frac{d \Phi(p)}{d m}=\frac{\Phi(p)}{M-m}>0
$$

$$
\begin{aligned}
& \frac{\partial}{\partial m} \eta(m, M(m))=-\varphi(p)+\omega \varphi(p)[M-m-2 m \Phi(p)-2 M(1-\Phi(p))- \\
& (M-m)(1-2 \Phi(p))]=-\varphi(p)+\omega \varphi(p)[2 m-2 M-4 m \Phi(p)+4 M \Phi(p)] .
\end{aligned}
$$

On the other hand,

$$
\begin{aligned}
& \frac{\partial}{\partial M} \eta(m, M(m))=\varphi(p)-\omega \varphi(p)[M-m-2 m \Phi(p)-2 M(1-\Phi(p))+ \\
& (M-m)(1-2(1-\Phi(p)))]=-\varphi(p)+\omega \varphi(p)[2 m-2 M-4 m \Phi(p)+4 M \Phi(p)] .
\end{aligned}
$$

So that

$$
\frac{d M}{d m}=1>0 \text {. }
$$

Putting these results together, the change in the second-best cost of a contract following a marginal change in $m$ is:

$$
\frac{d}{d m} E[W(\tilde{\pi})]=\Phi(p)-(M-m) \frac{\Phi(p)}{M-m}+\frac{d M}{d m}(1-\Phi(p))=1-\Phi(p)>0,
$$

which is positive, so any contract with $m>L$ is dominated. Therefore, at the optimum, $m=L$. 


\section{Proof of Proposition 4:}

We will demonstrate that if $L$ is negative and arbitrarily small, if $c, e^{*}$ and $\omega$ are bounded away from zero, and if $c e^{*}$ is bounded from above, then the Mirrlees approximation of the optimal contract (which is obtained in a setting without any lower bound on payments) is the optimal contract, and the agency cost is approximately zero.

In the first part of the proof, we show in four steps that $m=L, M \rightarrow_{L \rightarrow-\infty} w^{*}$, $p \rightarrow \rightarrow_{L \rightarrow-\infty}-\infty$.

First, we know from Proposition 3 that the optimal contract is characterized by $m=L$. Second, since the variance of $W(\tilde{\pi})$ is positive,

$$
-\omega\left[(m-E[W(\tilde{\pi})])^{2} \Phi(p)+(M-E[W(\tilde{\pi})])^{2}(1-\Phi(p))\right]<0 .
$$

For the participation constraint already written in (29) to be satisfied, we therefore need to have:

$$
m \Phi(p)+M(1-\Phi(p))>\bar{U}+c \frac{e^{*}}{2}=w^{*} .
$$

Since $m$ is arbitrarily small, $0<\Phi(p)<1$, and $0<1-\Phi(p)<1$, this inequality requires that $M>w^{*}$. For any given $M$, let $\varepsilon$ be implicitly defined by $M \equiv w^{*}+\varepsilon$.

Third, for a negative and arbitrarily small $L$, given that $\omega$ is bounded away from zero and that $M>w^{*}$, all terms on the left-hand side of the incentive constraint (27) are negligible next to $\varphi(p) L^{2} \omega$. We must therefore have $\varphi(p) L^{2} \omega \approx c e^{*}$ for the incentive constraint to be satisfied. This rewrites as:

$$
\varphi(p) \rightarrow_{L \rightarrow-\infty} \frac{c e^{*}}{\omega} \frac{1}{L^{2}}
$$

Because $L$ is arbitrarily small, and $\omega$ is bounded away from zero, the right-hand side of (35) tends to zero as $L$ approaches minus infinity. Since $\varphi(p)$ tends to zero as $p$ approaches minus infinity, (35) is verified if:

$$
p \rightarrow \rightarrow_{L \rightarrow-\infty}-\infty \text {. }
$$

Fourth, the normal distribution is characterized by:

$$
\frac{\Phi(p)}{\varphi(p)} \rightarrow_{p \rightarrow-\infty} 0 .
$$

Rewrite the participation constraint in (29) as:

$$
\begin{aligned}
& L \Phi(p)+\left(w^{*}+\varepsilon\right)(1-\Phi(p))-\omega\left[(L-E[W(\tilde{\pi})])^{2} \Phi(p)+\right. \\
& \left.\left(w^{*}+\varepsilon-E[W(\tilde{\pi})]\right)^{2}(1-\Phi(p))\right]=w^{*} .
\end{aligned}
$$

Consider the first two terms on the left-hand side of (38): Substituting from (35) into (37):

$$
\frac{\Phi(p)}{c e^{*}} \omega L^{2} \rightarrow_{p \rightarrow-\infty} 0 .
$$


Since $\omega$ is bounded away from zero and $c e^{*}$ is bounded from above, this implies that:

$$
E[W(\tilde{\pi})]=L \Phi(p)+\left(w^{*}+\varepsilon\right)(1-\Phi(p)) \rightarrow_{p \rightarrow-\infty} w^{*}+\varepsilon .
$$

Consider the third term on the left-hand side of (38). Since $\omega$ is bounded away from zero and $c e^{*}$ is bounded from above, (39) also implies that:

$$
\Phi(p) L^{2} \rightarrow_{p \rightarrow-\infty} 0 .
$$

So that, using (40),

$$
\begin{aligned}
& (L-E[W(\tilde{\pi})])^{2} \Phi(p)+\left(w^{*}+\varepsilon-E[W(\tilde{\pi})]\right)^{2}(1-\Phi(p))= \\
& \left(L-w^{*}-\varepsilon\right)^{2} \Phi(p)+\left(w^{*}+\varepsilon-w^{*}-\varepsilon\right)^{2}(1-\Phi(p)) \rightarrow_{p \rightarrow-\infty} 0 .
\end{aligned}
$$

Combining (40) and (41), the participation constraint in (38) rewrites as:

$$
w^{*}+\varepsilon \rightarrow_{p \rightarrow-\infty} w^{*} .
$$

So that, given (36),

$$
\varepsilon \rightarrow_{L \rightarrow-\infty} 0 \text {. }
$$

and

$$
M \rightarrow_{L \rightarrow-\infty} w^{*} .
$$

The first part of proof is complete.

Using the definition of the agency cost in (6), we know from (40) that the agency cost with the proposed contract is equal to:

$$
L \Phi(p)+\left(w^{*}+\varepsilon\right)(1-\Phi(p))-w^{*} \rightarrow_{L \rightarrow-\infty} \varepsilon .
$$

Using (43), the agency cost is approximately zero for an arbitrarily small $L$, and the proof is complete.

\section{Proof of Corollary 1:}

Given a convex contract $f$, we construct the corresponding concave contract $h$ as in the Proof of Proposition 1. Using the same lines as in Proof 1, we get:

$$
\begin{gathered}
\operatorname{var}\left[h(\tilde{\pi}) \mid e^{*}\right]=\operatorname{var}\left[f(\tilde{\pi}) \mid e^{*}\right], \\
\frac{\partial}{\partial e} \operatorname{var}\left[f(\tilde{\pi}) \mid e^{*}\right]>0, \quad \text { and } \frac{\partial}{\partial e} \operatorname{var}\left[h(\tilde{\pi}) \mid e^{*}\right]<0 .
\end{gathered}
$$

Moreover,

$$
\begin{aligned}
& \frac{d}{d e}\left\{E\left[f(\tilde{\pi}) \mid e^{*}\right]\right\}=E\left[(1+\gamma \tilde{\varepsilon}) f^{\prime}(\tilde{\pi}) \mid e^{*}\right]=\gamma \operatorname{cov}\left(\tilde{\varepsilon}, f^{\prime}(\tilde{\pi})\right)+E\left[(1+\gamma \tilde{\varepsilon}) \mid e^{*}\right] E\left[f^{\prime}(\tilde{\pi}) \mid e^{*}\right] . \\
& \frac{d}{d e}\left\{E\left[h(\tilde{\pi}) \mid e^{*}\right]\right\}=E\left[(1+\gamma \tilde{\varepsilon}) h^{\prime}(\tilde{\pi}) \mid e^{*}\right]=\gamma \operatorname{cov}\left(\tilde{\varepsilon}, h^{\prime}(\tilde{\pi})\right)+E\left[(1+\gamma \tilde{\varepsilon}) \mid e^{*}\right] E\left[h^{\prime}(\tilde{\pi}) \mid e^{*}\right] .
\end{aligned}
$$


By construction, we have as before $E\left[f^{\prime}(\tilde{\pi}) \mid e^{*}\right]=E\left[h^{\prime}(\tilde{\pi}) \mid e^{*}\right]$, so that the second terms on the right-hand-sides of (45) and (46) take the same value. In addition, with an initial convex contract $\left(f^{\prime \prime}>0\right)$, we have $\operatorname{cov}\left(\tilde{\varepsilon}, f^{\prime}(\tilde{\pi})\right)>0$ (indeed, $\frac{d f^{\prime}(e+e \varepsilon+\gamma e \varepsilon)}{d \varepsilon}=(1+\gamma e) f^{\prime \prime}(\pi)$, which is of the same sign as $f^{\prime \prime}$ given that $\left.\gamma \in\left(-1 / e^{*}, 0\right)\right)$. As $h$ is concave by construction $\left(h^{\prime \prime}<0\right)$, we have $\operatorname{cov}\left(\tilde{\varepsilon}, h^{\prime}(\tilde{\pi})\right)<0$. With $\gamma \in\left(-e^{*}, 0\right)$, it follows that:

$$
\frac{d}{d e}\left\{E\left[h(\tilde{\pi}) \mid e^{*}\right]\right\}>\frac{d}{d e}\left\{E\left[f(\tilde{\pi}) \mid e^{*}\right]\right\} .
$$

The rest of the proof follows the same lines as the Proofs of Propositions 1 and 2 above.

\section{Proof of Proposition 5:}

Given a concave contract $f$, we construct the corresponding convex contract $h$ as in the Proof of Proposition 1. Using the same lines as in Proof 1, we get:

$$
\begin{gathered}
\operatorname{var}\left[h(\tilde{\pi}) \mid e^{*}\right]=\operatorname{var}\left[f(\tilde{\pi}) \mid e^{*}\right], \\
\frac{\partial}{\partial e} \operatorname{var}\left[f(\tilde{\pi}) \mid e^{*}\right]<0, \text { and } \frac{\partial}{\partial e} \operatorname{var}\left[h(\tilde{\pi}) \mid e^{*}\right]>0 .
\end{gathered}
$$

Moreover, (remember that $f^{\prime \prime}<0$ and $h^{\prime \prime}>0$ now, so that the inequalities are reversed).

$$
\begin{aligned}
& \frac{d}{d e}\left\{E\left[f(\tilde{\pi}) \mid e^{*}\right]\right\}=E\left[(1+\gamma \tilde{\varepsilon}) f^{\prime}(\tilde{\pi}) \mid e^{*}\right]=\gamma \operatorname{cov}\left(\tilde{\varepsilon}, f^{\prime}(\tilde{\pi})\right)+E\left[(1+\gamma \tilde{\varepsilon}) \mid e^{*}\right] E\left[f^{\prime}(\tilde{\pi}) \mid e^{*}\right] . \\
& \frac{d}{d e}\left\{E\left[h(\tilde{\pi}) \mid e^{*}\right]\right\}=E\left[(1+\gamma \tilde{\varepsilon}) h^{\prime}(\tilde{\pi}) \mid e^{*}\right]=\gamma \operatorname{cov}\left(\tilde{\varepsilon}, h^{\prime}(\tilde{\pi})\right)+E\left[(1+\gamma \tilde{\varepsilon}) \mid e^{*}\right] E\left[h^{\prime}(\tilde{\pi}) \mid e^{*}\right] .
\end{aligned}
$$

By construction, we have as before $E\left[f^{\prime}(\tilde{\pi}) \mid e^{*}\right]=E\left[h^{\prime}(\tilde{\pi}) \mid e^{*}\right]$, so that the second terms on the right-hand-sides of (48) and (49) take the same value. In addition, with an initial concave contract $\left(f^{\prime \prime}<0\right)$, we have $\operatorname{cov}\left(\tilde{\varepsilon}, f^{\prime}(\tilde{\pi})\right)<0$. As $h$ is convex by construction $\left(h^{\prime \prime}>0\right)$, we have $\operatorname{cov}\left(\tilde{\varepsilon}, h^{\prime}(\tilde{\pi})\right)>0$. With $\gamma>0$, it follows that:

$$
\frac{d}{d e}\left\{E\left[h(\tilde{\pi}) \mid e^{*}\right]\right\}>\frac{d}{d e}\left\{E\left[f(\tilde{\pi}) \mid e^{*}\right]\right\} .
$$

The contract $h$ provides more effort incentives than the contract $f$ if and only if:

$$
\frac{d}{d e}\left\{E\left[h(\tilde{\pi}) \mid e^{*}\right]\right\}-\omega \frac{d}{d e}\left\{\operatorname{var}\left(h(\tilde{\pi}) \mid e^{*}\right)\right\}>\frac{d}{d e}\left\{E\left[f(\tilde{\pi}) \mid e^{*}\right]\right\}-\omega \frac{d}{d e}\left\{\operatorname{var}\left(f(\tilde{\pi}) \mid e^{*}\right)\right\} .
$$

With $\omega \rightarrow 0$, given (50) this condition is satisfied. The rest of the proof follows the same lines as the Proofs of Propositions 1 and 2 above.

Pierre CHAIGNEAU is an Assistant Professor of Finance at HEC Montreal (Canada) and Research Associate at the Financial Markets Group of the LSE (UK). He holds a PhD in Finance from the London School of Economics. His research interests include corporate finance, agency theory, and the structure of compensation contracts. 